\title{
Advances in Molecular Characterization and Targeted Therapy in Dermatofibrosarcoma Protuberans
}

\author{
Piotr Rutkowski, ${ }^{1}$ Agnieszka Wozniak, ${ }^{2}$ and Tomasz Switaj ${ }^{1}$ \\ ${ }^{1}$ Department of Soft Tissue/Bone Sarcoma and Melanoma, Maria Sklodowska-Curie Memorial Cancer Center and \\ Institute of Oncology, Roentgena 5, 02-781 Warsaw, Poland \\ ${ }^{2}$ Laboratory of Experimental Oncology, Department of General Medical Oncology, Catholic University Leuven and \\ University Hospitals Leuven, 3000 Leuven, Belgium
}

Correspondence should be addressed to Piotr Rutkowski, rutkowskip@coi.waw.pl

Received 12 August 2010; Accepted 31 January 2011

Academic Editor: H. Kovar

Copyright (C) 2011 Piotr Rutkowski et al. This is an open access article distributed under the Creative Commons Attribution License, which permits unrestricted use, distribution, and reproduction in any medium, provided the original work is properly cited.

\begin{abstract}
The molecular pathogenesis of dermatofibrosarcoma protuberans (DFSP) involves distinctive rearrangement of chromosomes 17 and 22 leading to formation of the COL1A1-PDGFB fusion gene. The knowledge of molecular events underlying development of DFSP resulted in the implementation of targeted therapy with imatinib-a tyrosine kinase inhibitor (TKI), to the clinical practice. The striking efficacy of imatinib in advanced cases of DFSP has been demonstrated in a few clinical trials. Thus, imatinib is currently considered the gold standard in the treatment of inoperable and/or metastatic and/or recurrent cases of DFSP. Therapy with imatinib may potentially facilitate resection or decrease possible disfigurement related to radical surgical procedure. Following partial response on imatinib significant percentage of patients may be rendered free of the disease by surgery of the residual tumor.
\end{abstract}

\section{Introduction}

Dermatofibrosarcoma protuberans (DFSP) is a rare cutaneous-origin sarcoma with usually indolent growth (over years) and low metastatic potential. Regional/distant metastases probability is less than $5 \%[1,2]$. Metastases develop more commonly in DFSP-containing areas of high-grade fibrosarcoma-fibrosarcomatous-DFSP (DFSP-FS) [3-6], which is characterized by more aggressive course. If distant metastases occur they are often restricted to lungs, and less commonly to lymph nodes. The standard treatment of the localized disease is radical, wide local excision. It is recommended that margins of the surgical excision should exceed $2-3 \mathrm{~cm}[1,7]$. This procedure often requires application of reconstructive techniques and may result in cosmetic disfigurement or functional impairment. Unfortunately, the microscopically infiltrating pattern of tumor growth might lead to high rates of unexpected positive margins. Local recurrences may occur late, and they have been reported within the range of $24-90 \%$ [1, 3, 8-14]. Nevertheless, several reports provided data demonstrating lower frequency of recurrence rate [15-17]. Recurrent disease is more challenging surgically, due to tumor fixation to deeper structures. Microscopic infiltrations spreading from the tumor might also lead to high probability of unexpected nonradical resection. There is only limited experience with Mohs micrographic surgery in the treatment of localized DFSP [18-20].

\section{Molecular Pathogenesis}

DFSP is characterized by the presence of distinctive, reciprocal rearrangement of chromosomes 17 and 22 in the form of translocation $\mathrm{t}(17 ; 22)(\mathrm{q} 22 ; \mathrm{q} 13)$ or supernumerary ring chromosomes containing material from chromosomal regions $17 \mathrm{q} 22$ and $22 \mathrm{q} 13$ [21-31]. The rearrangement leads to the fusion of alpha chain type a (COL1A1) localized on $17 \mathrm{q} 22$ to the platelet-derived growth factor beta (PDGFB) localized on 22q13 (Figure 1) [32].

The $P D G F B$ gene product is a growth factor that serves as a ligand for the transmembrane receptor kinase PDGFRB. The formation of COL1A1-PDGFB fusion gene results in the constitutional upregulation of $P D G F B$ expression, leading 


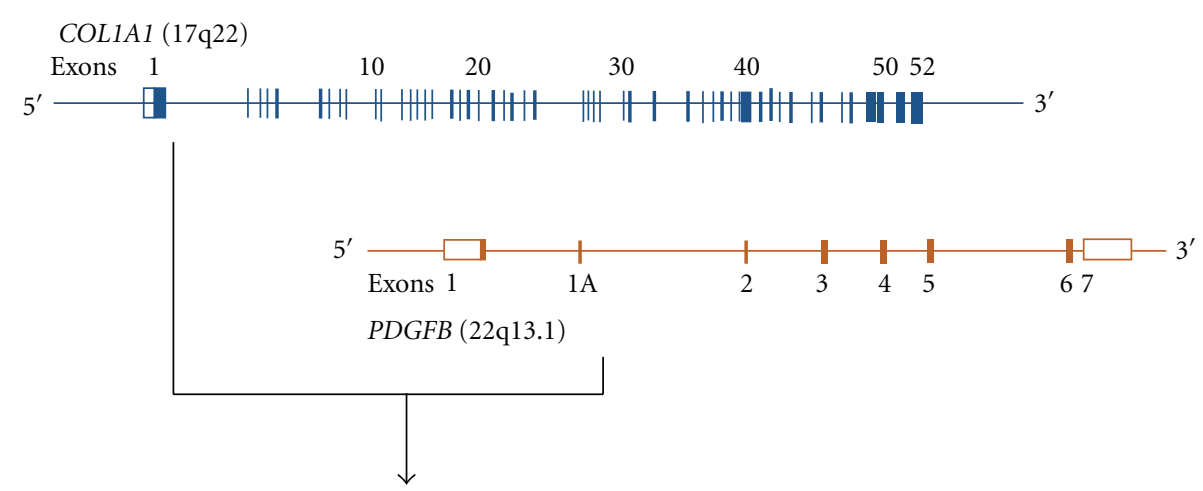

$\mathrm{t}(17 ; 22)(\mathrm{q} 22 ; \mathrm{q} 13.1)$ or ring chromosome

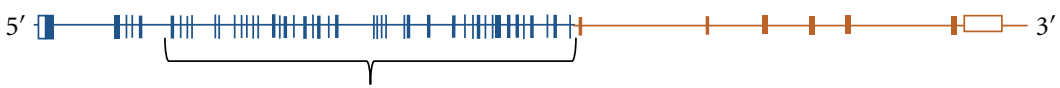

Breakpoint variability area (exons 6-47)

COL1A1-PDGFB fusion gene

FIGURE 1: Schematic presentation of the COL1A1/PDGFB fusion gene formation.

Normal chromosome 22

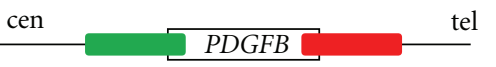

Derivative chromosome 17

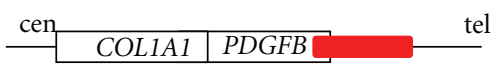

(a)

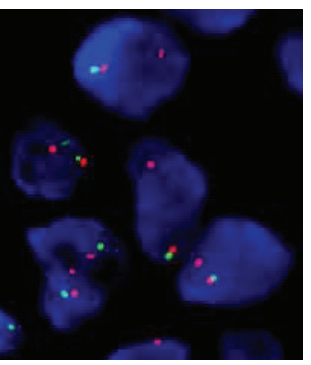

(b)

FIgURE 2: $P D G F B$ break-apart FISH in interphase nuclei from DFSP. (a) Schematic localization of FISH probes; (b) PDGFB rearrangement detected by FISH, evidenced by one copy (red probe) of the telomeric PDGFB signal in tumor cells (courtesy of Professor M. Debiec-Rychter).

to continuous autocrine activation of PDGF receptor $B$ (PDGFRB) and as a consequence to propagation of the mitotic signal by formation of an autocrine and paracrine loops [33-35]. Greco et al. [36, 37] provided evidence, that transfection with COL1A1-PDGFB fusion gene could transform NIH3T3 cells. Furthermore, it was shown that by using suramin, a compound known to interfere with PDGFPDGFR ligand-receptor interaction, the COL1A1-PDGFB transformed phenotype in NIH3T3 cells can be reversed [36].

Interestingly the presence of the specific COL1A1$P D G F B$ fusion transcript was also identified in giant cell fibroblastoma (GCF) that is a histologic variant of DFSP. GCF primarily affects children so it is also called the juvenile form of DFSP [38-41]. In DFSP-FS increased copy numbers of COL1A1-PDGFB fusion gene were observed suggesting a possible oncogenic mechanism of the clonal evolution from DFSP into DFSP-FS [42].

Although there is no need for molecular confirmation of the diagnosis in the majority of DFSP cases, the detection of the chromosomal 17;22 rearrangements or the COL1A1-PDGFB fusion is a valuable diagnostic tool for differential diagnosis of atypical, metastatic DFSP or DFSPFS. Currently two main molecular techniques are used: fluorescence in situ hybridization (FISH) or multiplex reverse transcription polymerase chain reaction (RT-PCR). FISH can be performed on interphase nuclei from cell suspensions, touch prints, or frozen or fixed paraffin-embedded sections most commonly using break-apart PDGFB or COL1A1$P D G F B$ fusion approach (Figure 2). On the other hand, RTPCR requires RNA extracted from tumor fragments and necessitates the simultaneous use of several COL1A1 primers (multiplex approach) as the breakpoint can randomly occur between exons 6 and 47 [43-45].

\section{Targeted Therapy}

Advances in the understanding of molecular mechanisms of DFSP resulted in the implementation of targeted therapy based on PDGFR inhibition to the treatment of this sarcoma. Imatinib mesylate is a tyrosine kinase inhibitor rationally developed and specifically directed against BCR/ABL, KIT, FMS (receptor for Colony Stimulating Factor 1), ARG (ABL-related gene), and PDGFR alpha and beta. It has been also found to be the first effective systemic therapy in DFSP. Imatinib competes with adenosine triphosphate (ATP) molecule, blocking tyrosine kinase receptor ability for autophosphorylation, which in return results in inhibition of the aberrant signal transduction pathway and partial restoration of proper intracellular signaling. The observation that autocrine overproduction of PDGFB caused by gene rearrangement is a key pathogenetic factor $[33,34]$ forced the in vitro research, which showed inhibition of DFSP cells growth in vitro after exposure to imatinib [36, 46]. The 


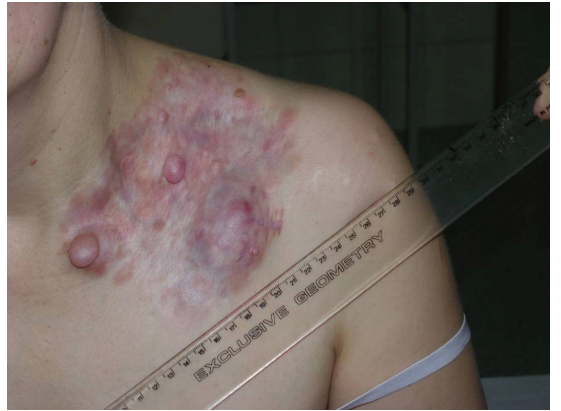

(a)

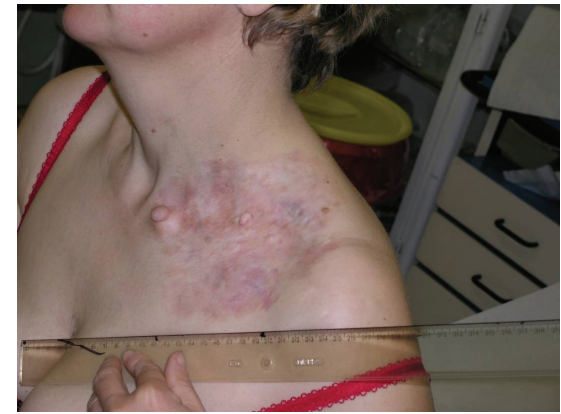

(b)

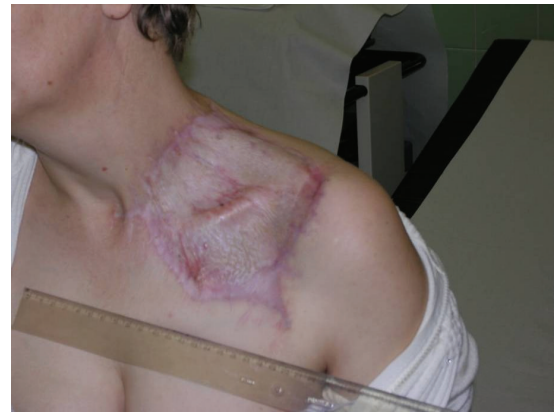

(c)

FIGURE 3: Images of advanced dermatofibrosarcoma protuberans of the supraclavicular region before and after therapy with imatinib, and after resection of residual disease. The patient is now 3 years free of disease.

further demonstration of the imatinib inhibitory effect on six different DFSP cell lines both in vitro and in vivo [37] has led to the investigation of this new therapeutic approach in the clinic. Early case reports on small series of patients suggested the usefulness of imatinib in metastatic and locally advanced DFSP [47-52]. Next series of 10 patients with locally advanced and/or metastatic DFSP treated within Imatinib Target Exploration Consortium Study B2225 showed responses in all patients, including complete responses in five out of 10 of locally advanced cases and one partial response lasting seven months in metastatic case [53]. As a consequence imatinib was registered as a therapy of choice in advanced (inoperable and/or metastatic) DFSPs (Figure 3). In a phase II trial [54] evaluating the activity of imatinib in life-threatening malignancies expressing imatinib-sensitive tyrosine kinases DFSP was the only one of five tumor types in which a notable activity was shown including extensive regression in 10/20 cases (50\% partial remissions, 33,3\% complete remissions).

Combined analysis of prematurely closed, two phase II, single arm, open-label trials on efficacy of imatinib in advanced DFSP (European Organisation for Research and Treatment of Cancer no. 62027 and the Southwest Oncology Group no. S0345) has demonstrated the clinical benefit with rate exceeding $70 \%$ and median time to progression of 1.7 years on 25 patients with advanced DFSP [55]. Although there were some differences in both trials' design, the observed responses' rates were similar. These results imply that the imatinib dose of $400 \mathrm{mg}$ daily has similar efficacy to $800 \mathrm{mg}$ daily in this entity. Rutkowski et al. [56] have proved striking activity of imatinib mesylate in advanced DFSP in the group of 15 patients treated with imatinib in routine clinical practice outside any trial, with clinical benefit rate approaching $80 \%$ as well as median PFS and OS being not reached. In Table 1 the efficacy results of imatinib in advanced DFSPs from pooled analysis of phase II trials [55] and 15 patients treated outside clinical trials is presented [56].

It has also been shown that DFSPs-FS with $t(17 ; 22)$ are still imatinibsensitive although responses seem to last shorter [57] while DFSPs-FS lacking the specific aberration do not
TABLE 1: The best overall responses, progression, and survival status in combined phase II clinical trials [55] and in group of patients treated outside clinical trials [56].

\begin{tabular}{lcc}
\hline & $\begin{array}{c}\text { Group of 24 } \\
\text { patients treated in } \\
\text { phase II trials [55] }\end{array}$ & $\begin{array}{c}\text { Group of 15 patients } \\
\text { treated outside } \\
\text { clinical trials [56] }\end{array}$ \\
\hline $\begin{array}{c}\text { Progression status } \\
\text { Progression-free }\end{array}$ & $12(50)$ & $11(73)$ \\
Progression & $12(50)$ & $4(27)$ \\
Survival status & $18(75)$ & $12(80)$ \\
Alive & $6(25)$ & $3(20)$ \\
Dead & & $11(73)$ \\
Best overall response & $11(45.9)$ & $1(7)$ \\
Partial response & $6(25)$ & $3(20)$ \\
Stable disease & $4(16.6)$ & 0 \\
Progressive disease & $3(12.5)$ & \\
Not evaluable & & \\
\hline
\end{tabular}

respond to the treatment [53]. Therefore the confirmation of the molecular target (COL1A1-PDGFB fusion) presence seems to be obligatory in every case prior to the start of imatinib therapy.

Complete, wide surgical excision is the standard treatment in localized, resectable cases, and in advanced cases it may result in cosmetic disfigurement or serious functional impairment. Thus the neoadjuvant imatinib strategy leading to tumor downstaging and decrease of excision morbidity by tissue-sparing appears to be very attractive. Kérob et al. [58] presented report on 25 resectable DFSP (median size: $4.5 \mathrm{~cm}$ ) treated in phase II trial with preoperative imatinib at the dose of $600 \mathrm{mg}$ daily for two months. The objective partial response according to RECIST was observed in nine cases $(36 \%)$. The median relative tumor volume decrease was 20\% (range: 12.5-100\%). Available clinical data indicate that some DFSP patients initially evaluated as 
unresectable/metastatic or necessitating mutilating surgery turned out to have resectable tumor after imatinib therapy. This rational approach enabling achievement of complete remission may be potentially curative, although longer followup is needed. Further studies are required for elucidating whether preoperative imatinib therapy reduces the need for wide surgical margins or whether imatinib has activity as adjuvant therapy in cases with positive margins after excision or in other high-risk patients.

Majority of patients treated with imatinib experienced side effects during treatment, but almost all are mild and manageable. The most common were fluid retention/edema, anemia, fatigue, nausea, skin rash, thrombocytopenia, vomiting, neutropenia, and diarrhea, and they are similar to those observed in patients with gastrointestinal stromal tumor (GIST).

There are still several questions regarding imatinib mechanism of action, and possible resistance to this targeted therapy in DFSP. There is also a need to identify novel predictive molecular markers for patients' outcome. It was presumed that imatinib effect resulted from inhibition of PDGFR phosphorylation. Surprisingly, clinical activity of imatinib in DFSP is striking even in DFSP expressing relatively low amounts of activated receptor. It seems that inhibition of low-level receptor tyrosine kinase may be effective clinically if tumor cells are dependent on that signaling mechanism, what has been observed also in pigmented villonodular synovitis/tenosynovial giant-cell tumor $[59,60]$. The better understanding of the downstream effects caused by imatinib-PDGFB interaction would allow defining additional treatment strategies for DFSP patients. In case of disease progression after initial response to imatinib the investigation of other multitargeted tyrosine kinase inhibitors seems to be justified.

To summarize, imatinib therapy is currently the gold standard in the treatment of inoperable and/or metastatic and/or recurrent cases of DFSP, and this targeted therapy may potentially facilitate resection or decrease possible disfigurement. Significant percentage of patients may be rendered free of disease by surgery of residual disease following partial imatinib responses. Current therapy of DFSP with $t(17 ; 22)$ translocation should be conducted by multidisciplinary team, including oncological surgeon. The use of imatinib mesylate as initial therapy should be considered to decrease possible extent of surgery and related morbidity.

\section{References}

[1] C. K. Chang, I. A. Jacobs, and G. I. Salti, "Outcomes of surgery for dermatofibrosarcoma protuberans," European Journal of Surgical Oncology, vol. 30, no. 3, pp. 341-345, 2004.

[2] W. B. Laskin, "Dermatofibrosarcoma protuberans," CA: A Cancer Journal for Clinicians, vol. 42, no. 2, pp. 116-125, 1992.

[3] W. B. Bowne, C. R. Antonescu, D. H. Y. Leung et al., "Dermatofibrosarcoma protuberans: a clinicopathologic analysis of patients treated and followed at a single institution," Cancer, vol. 88 , no. 12 , pp. 2711-2720, 2000.
[4] T. Mentzel, A. Beham, D. Katenkamp, A. P. Dei Tos, and C. D. M. Fletcher, "Fibrosarcomatous ('high-grade') dermatofibrosarcoma protuberans: clinicopathologic and immunohistochemical study of a series of 41 cases with emphasis on prognostic significance," American Journal of Surgical Pathology, vol. 22, no. 5, pp. 576-587, 1998.

[5] P. Lal, R. Sharma, H. Mohan, and M. S. Sekhon, "Dermatofibrosarcoma protuberans metastasizing to lymph nodes: a case report and review of literature," Journal of Surgical Oncology, vol. 72, no. 3, pp. 178-180, 1999.

[6] C. Diaz-Cascajo, W. Weyers, L. Bornego et al., "Dermatofibrosarcoma protuberans with fibrosarcomatous areas: a clinico-pathologic and immunohistochemical study four cases," American Journal of Dermathopathology, vol. 19, pp. 562-567, 1997.

[7] Z. Kimmel, D. Ratner, J. Y. S. Kim, J. D. Wayne, A. W. Rademaker, and M. Alam, "Peripheral excision margins for dermatofibrosarcoma protuberans: a meta-analysis of spatial data," Annals of Surgical Oncology, vol. 14, no. 7, pp. 21132120, 2007.

[8] H. M. Gloster, K. R. Harris, and R. K. Roenigk, "A comparison between Mohs micrographic surgery and wide surgical excision for the treatment of dermatofibrosarcoma protuberans," Journal of the American Academy of Dermatology, vol. 35, no. 1, pp. 82-87, 1996.

[9] L. Barnes, J. A. Coleman Jr., and J. T. Johnson, "Dermatofibrosarcoma protuberans of the head and neck," Archives of Otolaryngology, vol. 110, no. 6, pp. 398-404, 1984.

[10] D. F. Roses, Q. Valensi, G. LaTrenta, and M. N. Harris, "Surgical treatment of dermatofibrosarcoma protuberans," Surgery Gynecology and Obstetrics, vol. 162, no. 5, pp. 449-452, 1986.

[11] E. J. Th. Rutgers E.J., B. B. R. Kroon, C. E. Albus-Lutter, and E. Gortzak, "Dermatofibrosarcoma protuberans: treatment and prognosis," European Journal of Surgical Oncology, vol. 18, no. 3, pp. 241-248, 1992.

[12] C. K. Koh, C. B. Ko, H. P. R. Bury, and E. H. Wyatt, "Dermatofibrosarcoma protuberans," International Journal of Dermatology, vol. 34, no. 4, pp. 256-260, 1995.

[13] A. Stojadinovic, H. M. Karpoff, C. R. Antonescu et al., "Dermatofibrosarcoma protuberans of mthe head and neck," Annals of Surgical Oncology, vol. 7, no. 9, pp. 696-704, 2000.

[14] S. T. Heuvel, A. Suurmeijer, E. Pras, R. J. Van Ginkel, and H. J. Hoekstra, "Dermatofibrosarcoma protuberans: recurrence is related to the adequacy of surgical margins," European Journal of Surgical Oncology, vol. 36, no. 1, pp. 89-94, 2010.

[15] M. Fiore, R. Miceli, C. Mussi et al., "Dermatofibrosarcoma protuberans treated at a single institution: a surgical disease with a high cure rate," Journal of Clinical Oncology, vol. 23, no. 30, pp. 7669-7675, 2005.

[16] D. DuBay, V. Cimmino, L. Lowe, T. M. Johnson, and V. K. Sondak, "Low recurrence rate after surgery for dermatofibrosarcoma protuberans: a multidisciplinary approach from a single institution," Cancer, vol. 100, no. 5, pp. 1008-1016, 2004.

[17] J. M. Farma, J. B. Ammori, J. S. Zager et al., "Dermatofibrosarcoma protuberans: how wide should we resect?" Annals of Surgical Oncology, vol. 17, pp. 2112-2118, 2010.

[18] J. Wacker, B. Khan-Durani, and W. Hartschuh, "Modified mohs micrographic surgery in the therapy of dermatofibrosarcoma protuberans: analysis of 22 patients," Annals of Surgical Oncology, vol. 11, no. 4, pp. 438-444, 2004. 
[19] V. P. Khatri, J. M. Galante, R. J. Bold, P. D. Schneider, R. Ramsamooj, and J. E. Goodnight, "Dermatofibrosarcoma protuberans: reappraisal of wide local excision and impact of inadequate initial treatment," Annals of Surgical Oncology, vol. 10, no. 9, pp. 1118-1122, 2003.

[20] A. N. Meguerditchian, J. Wang, B. Lema, W. G. Kraybill, N. C. Zeitouni, and J. M. Kane, "Wide excision or Mohs micrographic surgery for the treatment of primary dermatofibrosarcoma protuberans," American Journal of Clinical Oncology, vol. 33, no. 3, pp. 300-303, 2010.

[21] J. A. Bridge, J. R. Neff, and A. A. Sandberg, "Cytogenetic analysis of dermatofibrosarcoma protuberans," Cancer Genetics and Cytogenetics, vol. 49, no. 2, pp. 199-202, 1990.

[22] N. Mandahl, S. Heim, H. Willen, A. Rydholm, and F. Mitelman, "Supernumerary ring chromosome as the sole cytogenetic abnormality in a dermatofibrosarcoma protuberans," Cancer Genetics and Cytogenetics, vol. 49, no. 2, pp. 273275, 1990.

[23] N. Mandahl, J. Limon, F. Mertens, K. Arheden, and F. Mitelman, "Ring marker containing $17 \mathrm{q}$ and chromosome 22 in a case of dermatofibrosarcoma protuberans," Cancer Genetics and Cytogenetics, vol. 89, no. 1, pp. 88-91, 1996.

[24] F. Pedeutour, J. M. Coindre, G. Sozzi et al., "Supernumerary ring chromosomes containing chromosome 17 sequences: a specific feature of dermatofibrosarcoma protuberans?" Cancer Genetics and Cytogenetics, vol. 76, no. 1, pp. 1-9, 1994.

[25] F. Pedeutour, M. P. Simon, F. Minoletti et al., "Ring 22 chromosomes in dermatofibrosarcoma protuberans are lowlevel amplifiers of chromosome 17 and 22 sequences," Cancer Research, vol. 55, no. 11, pp. 2400-2403, 1995.

[26] F. Pedeutour, J. P. Lacour, C. Perrin et al., "Another case of $\mathrm{t}(17 ; 22)(\mathrm{q} 22 ; \mathrm{q} 13)$ in an infantile dermatofibrosarcoma protuberans," Cancer Genetics and Cytogenetics, vol. 89, no. 2, pp. 175-176, 1996.

[27] F. Pedeutour, M. P. Simon, F. Minoletti et al., "Translocation, $\mathrm{t}(17 ; 22)(\mathrm{q} 22 ; \mathrm{q} 13)$, in dermatofibrosarcoma protuberans: a new tumor-associated chromosome rearrangement," Cytogenetics and Cell Genetics, vol. 72, no. 2-3, pp. 171-174, 1996.

[28] S. Kiuru-Kuhlefelt, W. El-Rifai, J. Fanburg-Smith, J. Kere, M. Miettinen, and S. Knuutila, "Concomitant DNA copy number amplification at $17 \mathrm{q}$ and $22 \mathrm{q}$ in dermatofibrosarcoma protuberans," Cytogenetics and Cell Genetics, vol. 92, no. 3-4, pp. 192-195, 2001.

[29] S. C. Linn, R. B. West, J. R. Pollack et al., "Gene expression patterns and gene copy number changes in dermatofibrosarcoma protuberans," American Journal of Pathology, vol. 163, no. 6, pp. 2383-2395, 2003.

[30] A. A. Sandberg and J. A. Bridge, "Updates on the cytogenetics and molecular genetics of bone and soft tissue tumors: dermatofibrosarcoma protuberans and giant cell fibroblastoma," Cancer Genetics and Cytogenetics, vol. 140, no. 1, pp. 1-12, 2003.

[31] N. Sirvent, G. Maire, and F. Pedeutour, "Genetics of dermatofibrosarcoma protuberans family of tumors: from ring chromosomes to tyrosine kinase inhibitor treatment," Genes Chromosomes and Cancer, vol. 37, no. 1, pp. 1-19, 2003.

[32] M. P. Simon, F. Pedeutour, N. Sirvent et al., "Deregulation of the platelet-derived growth factor B-chain gene via fusion with collagen gene COL1A1 in dermatofibrosarcoma protuberans and giant-cell fibroblastoma," Nature Genetics, vol. 15, no. 1, pp. 95-98, 1997.
[33] A. Shimizu, K. P. O’Brien, T. Sjöblom et al., “The dermatofibrosarcoma protuberans-associated collagen type $\mathrm{I} \alpha 1 /$ platelet-derived growth factor (PDGF) B-chain fusion gene generates a transforming protein that is processed to functional PDGF-BB," Cancer Research, vol. 59, no. 15, pp. 3719-3723, 1999.

[34] M.-P. Simon, M. Navarro, D. Roux et al., "Transforming properties of chimerical protein COL1A1-PDGFB generated by dermatofibrosarcoma protuberans-associated translocation t(17;22)(q22;q13.1)," Cancer Genetics and Cytogenetics, vol. 128 , p. 82, 2001.

[35] G. McArthur, "Molecularly targeted treatment for dermatofibrosarcoma protuberans," Seminars in Oncology, vol. 31, no. 6, pp. 30-36, 2004.

[36] A. Greco, L. Fusetti, R. Villa et al., "Transforming activity of the chimeric sequence formed by the fusion of collagen gene COL1A1 and the platelet derived growth factor b-chain gene in dermatofibrosarcoma protuberans," Oncogene, vol. 17, no. 10, pp. 1313-1319, 1998.

[37] A. Greco, E. Roccato, C. Miranda, L. Cleris, F. Formelli, and M. A. Pierotti, "Growth-inhibitory effect of STI571 on cells transformed by the COL1A1/PDGFB rearrangement," International Journal of Cancer, vol. 92, no. 3, pp. 354-360, 2001.

[38] P. Dal Cin, R. Sciot, I. De Wever et al., "Cytogenetic and immunohistochemical evidence that giant cell fibroblastoma is related to dermatofibrosarcoma protuberans," Genes Chromosomes and Cancer, vol. 15, no. 1, pp. 73-75, 1996.

[39] K. P. O’Brien, E. Seroussi, P. Dal Cin et al., "Various regions within the alpha-helical domain of the COL1A1 gene are fused to the second exon of the PDGFB gene in dermatofibrosarcomas and giant- cell fibroblastomas," Genes Chromosomes and Cancer, vol. 23, no. 2, pp. 187-193, 1998.

[40] M. J. Terrier-Lacombe, L. Guillou, G. Maire et al., "Dermatofibrosarcoma protuberans, giant cell fibroblastoma, and hybrid lesions in children: clinicopathologic comparative analysis of 28 cases with molecular data-a study from the french federation of cancer centers sarcoma group," American Journal of Surgical Pathology, vol. 27, no. 1, pp. 27-39, 2003.

[41] R. S. Macarenco, R. Zamolyi, M. F. Franco et al., "Genomic gains of COLIAI-PDFGB occur in the histologic evolution of giant cell fibroblastoma into dermatofibrosarcoma protuberans," Genes Chromosomes and Cancer, vol. 47, no. 3, pp. 260265, 2008.

[42] J. J. Abbott, M. Erickson-Johnson, X. Wang, A. G. Nascimento, and A. M. Oliveira, "Gains of COL1A1-PDGFB genomic copies occur in fibrosarcomatous transformation of dermatofibrosarcoma protuberans," Modern Pathology, vol. 19, no. 11, pp. 1512-1518, 2006.

[43] D. Kerob, F. Pedeutour, C. Leboeuf et al., "Value of cytogenetic analysis in the treatment of dermatofibrosarcoma protuberans," Journal of Clinical Oncology, vol. 26, no. 10, pp. 17571759, 2008.

[44] J. Wang, Y. Morimitsu, S. Okamoto et al., "COL1A1-PDGFB fusion transcripts in fibrosarcomatous areas of six dermatofibrosarcomas protuberans," Journal of Molecular Diagnostics, vol. 2, no. 1, pp. 47-52, 2000.

[45] K. U. Patel, S. S. Szabo, V. S. Hernandez et al., "Dermatofibrosarcoma protuberans COL1A1-PDGFB fusion is identified in virtually all dermatofibrosarcoma protuberans cases when investigated by newly developed multiplex reverse transcription polymerase chain reaction and fluorescence in situ hybridization assays," Human Pathology, vol. 39, no. 2, pp. 184-193, 2008. 
[46] T. Sjöblom, A. Shimizu, K. P. O’Brien et al., “Growth inhibition of dermatofibrosarcoma protuberans tumors by the platelet-derived growth factor receptor antagonist STI571 through induction of apoptosis," Cancer Research, vol. 61, no. 15, pp. 5778-5783, 2001.

[47] R. G. Maki, R. A. Awan, R. H. Dixon, S. Jhanwar, and C. R. Antonescu, "Differential sensitivity to imatinib of 2 patients with metastatic sarcoma arising from dermatofibrosarcoma protuberans," International Journal of Cancer, vol. 100, no. 6, pp. 623-626, 2002.

[48] B. P. Rubin, S. M. Schuetze, J. F. Eary et al., "Molecular targeting of platelet-derived growth factor B by imatinib mesylate in a patient with metastatic dermatofibrosarcoma protuberans," Journal of Clinical Oncology, vol. 20, no. 17, pp. 3586-3591, 2002.

[49] F. Pedeutour, J. M. Coindre, G. Nicolo et al., "Response of metastatic dermatofibrosarcoma protuberans to imatinib mesylate," Proceedings of the American Society of Clinical Oncology, vol. 23, abstract 3334, 2003.

[50] W. Ruka, S. Falkowski, U. Grzesiakowska et al., "The partial response of lung metastases arising from dermatofibrosarcoma protuberans after one month of imatinib mesylate therapy-a case report," Nowotwory, vol. 53, no. 2, pp. 165168, 2003.

[51] S. V. Labropoulos, J. A. Fletcher, A. M. Oliveira, S. Papadopoulos, and E. D. Razis, "Sustained complete remission of metastatic dermatofibrosarcoma protuberans with imatinib mesylate," Anti-Cancer Drugs, vol. 16, no. 4, pp. 461-466, 2005.

[52] K. Mizutani, Y. Tamada, K. Hara et al., "Imatinib mesylate inhibits the growth of metastatic lung lesions in a patient with dermatofibrosarcoma protuberans," British Journal of Dermatology, vol. 151, no. 1, pp. 235-237, 2004.

[53] G. A. McArthur, G. D. Demetri, A. Van Oosterom et al., "Molecular and clinical analysis of locally advanced dermatofibrosarcoma protuberans treated with imatinib: imatinib target exploration consortium study B2225," Journal of Clinical Oncology, vol. 23, no. 4, pp. 866-873, 2005.

[54] M. C. Heinrich, H. Joensuu, G. D. Demetri et al., "Phase II, open-label study evaluating the activity of imatinib in treating life-threatening malignancies known to be associated with imatinib- sensitivetyrosine kinases," Clinical Cancer Research, vol. 14, no. 9, pp. 2717-2725, 2008.

[55] P. Rutkowski, M. Van Glabbeke, C. J. Rankin et al., "Imatinib mesylate in advanced dermatofibrosarcoma protuberans: pooled analysis of two phase II clinical trials," Journal of Clinical Oncology, vol. 28, no. 10, pp. 1772-1779, 2010.

[56] P. Rutkowski, M. Dębiec-Rychter, Z. I. Nowecki et al., “Treatment of advanced dermatofibrosarcoma protuberans with imatinib mesylate with or without surgical resection," Journal of the European Academy of Dermatology and Venereology, vol. 25, no. 3, pp. 264-270, 2011.

[57] A. Gronchi, S. Stacchiotti, F. Pedeutour et al., "Response to imatinib mesylate (IM) in fibrosarcoma (FS) arising in dermatofibrosarcoma protuberans (DFSP)," Journal of Clinical Oncology, vol. 26, supplement, 2008, abstract 10593.

[58] D. Kérob, R. Porcher, O. Vérola et al., "Imatinib mesylate as a preoperative therapy in dermatofibrosarcoma: results of a multicenter phase II study on 25 patients," Clinical Cancer Research, vol. 16, no. 12, pp. 3288-3295, 2010.

[59] R. B. West, B. P. Rubin, M. A. Miller et al., "A landscape effect in tenosynovial giant-cell tumor from activation of CSF1 expression by a translocation in a minority of tumor cells," Proceedings of the National Academy of Sciences of the United States of America, vol. 103, no. 3, pp. 690-695, 2006.

[60] J. Y. Blay, H. El Sayadi, P. Thiesse, J. Garret, and I. RayCoquard, "Complete response to imatinib in relapsing pigmented villonodular synovitis/tenosynovial giant cell tumor (PVNS/TGCT)," Annals of Oncology, vol. 19, no. 4, pp. 821822, 2008. 


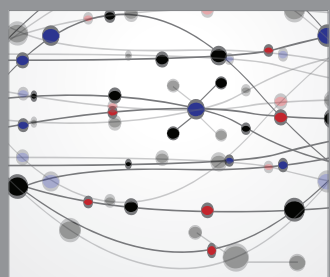

The Scientific World Journal
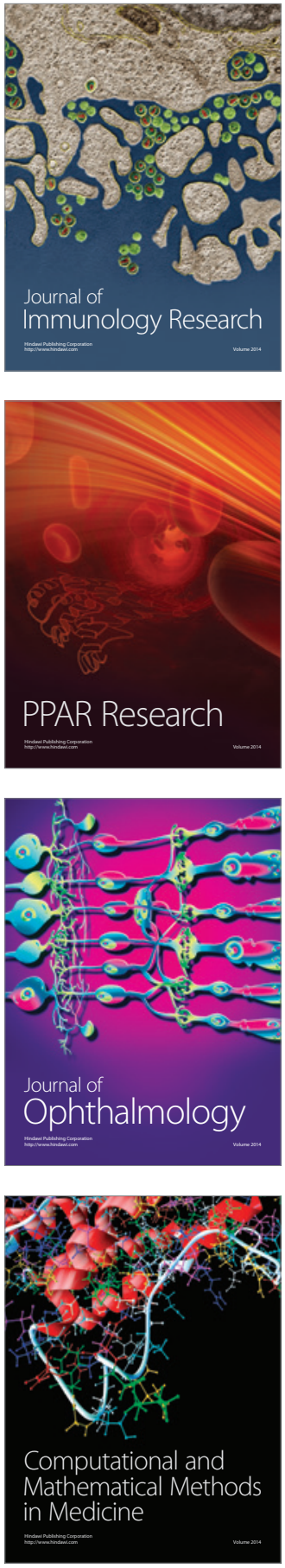

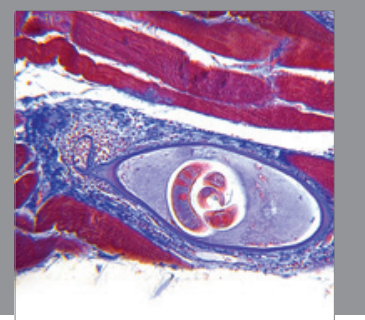

Gastroenterology

Research and Practice
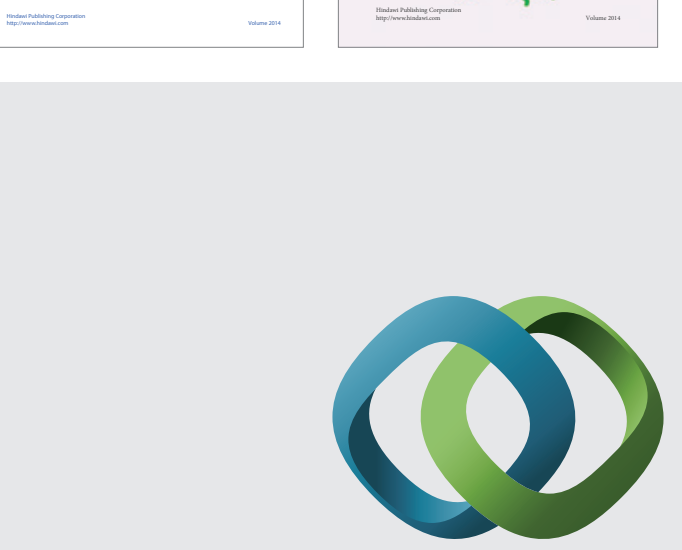

\section{Hindawi}

Submit your manuscripts at

http://www.hindawi.com
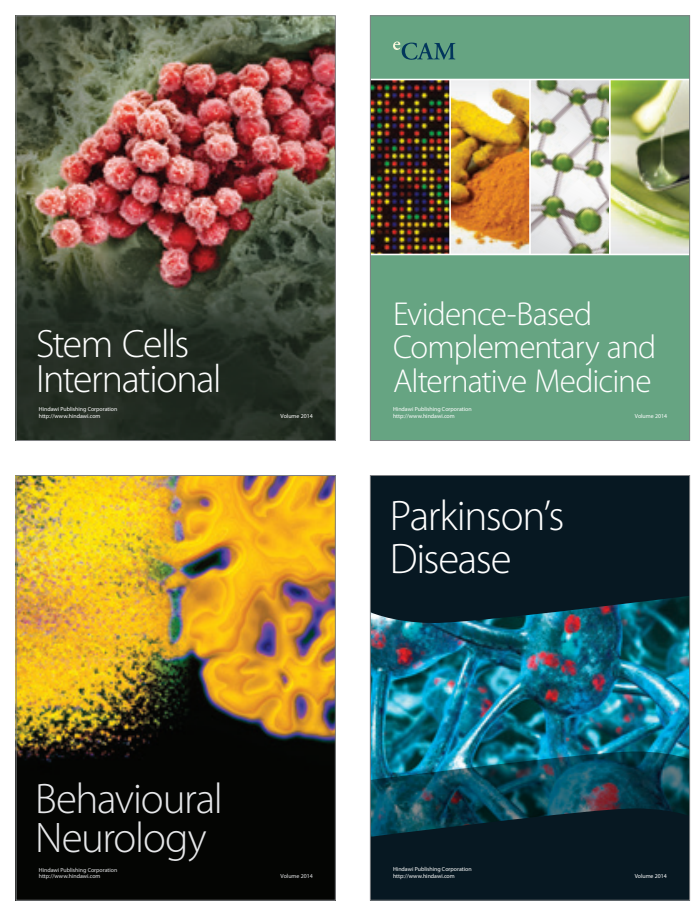

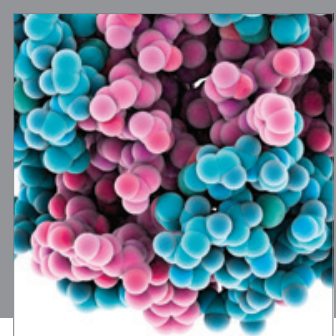

Journal of
Diabetes Research

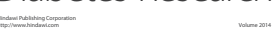

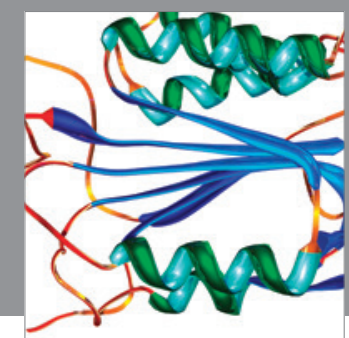

Disease Markers
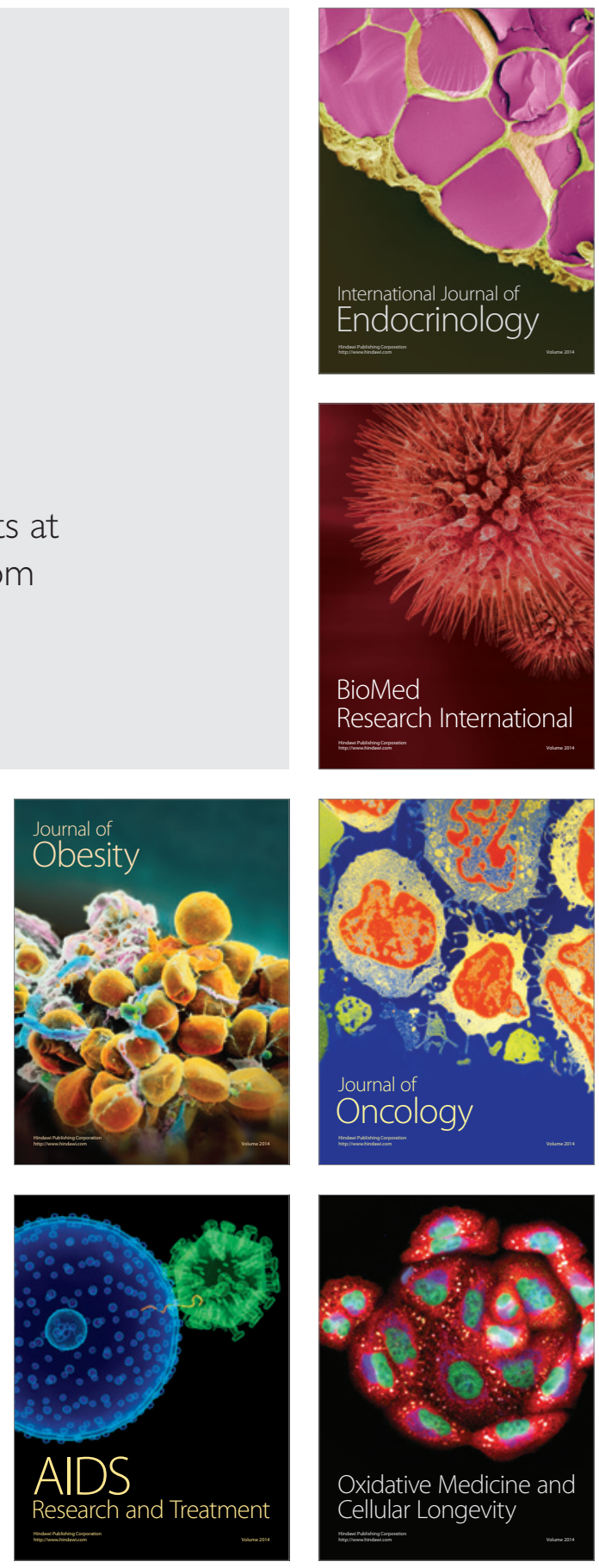